\title{
Impact of restriction of cefepime use on the antimicrobial susceptibility of Gram-negative bacilli related to healthcare-associated infections in an orthopedic hospital
}

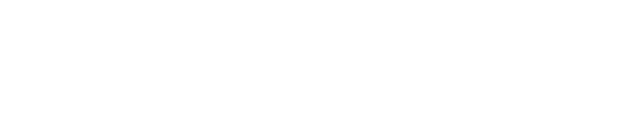

\author{
Priscila R Oliveira' \\ Adriana P Paula' \\ Karine Dal-Paz' \\ Cassia S Felix' \\ Flavia Rossi \\ Jorge S Silva' \\ Ana Lucia M Lima' \\ 'Orthopaedics and Traumatology \\ Department, ${ }^{2}$ Pathology Department, \\ University of São Paulo, São Paulo, \\ Brazil
}

\begin{abstract}
Introduction: In recent decades, antimicrobial resistance has become a public health problem, particularly in cases of healthcare-associated infections. Interaction between antibiotic consumption and resistance development is of particular interest regarding Gram-negative bacilli, whose growing resistance has represented a great challenge.

Objective: Assess the impact of restriction of cefepime use on antimicrobial susceptibility among the Gram-negative bacilli (GNB) most frequently involved in healthcare-associated infections (HAI).

Methods: Data relating to hospital occupancy and mortality rates, incidence of HAI, incidence of GNB as causative agents of HAI, antimicrobial consumption at the hospital and antimicrobial susceptibility of GNB related to HAI were compared between two periods: a 24-month period preceding restriction of cefepime use and a 24-month period subsequent to this restriction.

Results: There was a significant drop in cefepime consumption after its restriction. Susceptibility of Acinetobacter baumanii improved relating to gentamicin, but it worsened in relation to imipenem, subsequent to this restriction. For Pseudomonas aeruginosa, there was no change in antimicrobial susceptibility. For Klebsiella pneumoniae and Enterobacter spp, there were improvements in susceptibility relating to ciprofloxacin.

Conclusion: Restriction of cefepime use had a positive impact on K. pneumoniae and Enterobacter spp, given that after this restriction, their susceptibilities to ciprofloxacin improved. However, for A. baumanii, the impact was negative, given the worsening of susceptibility to imipenem.
\end{abstract}

Keywords: ecological studies, cross infection, drug resistance, microbial, Gram-negative bacilli, cefepime

\section{Introduction}

Over recent decades, resistance to antimicrobials has become a worldwide public health problem, and particularly important regarding bacterial agents that cause healthcare related infections. ${ }^{1}$ Today, there are bacteria responsible for infections in hospital environments that are resistant to all existing classes of antimicrobials, which shows their great potential for evolutionary adaptation. ${ }^{2}$ This, together with the declining numbers of new antimicrobials that are being certified and introduced into clinical practice, is making the growing prevalence of these bacteria with high levels of resistance a subject of increasing concern. ${ }^{3}$ The formerly abundant flow of new antibiotics of ever-broader spectrum supplied by the pharmaceutical industry has been shown to be a non-renewable resource. ${ }^{4}$
Correspondence: Priscila R Oliveira Rua Doutor Ovidio Pires de Campos, 333, sala 3 I IA, CEP 054030 I0,

São Paulo, SP, Brazil

Tel/Fax +55 II 30696900

Email priscilarosalba@gmail.com 
The options for attempting to control the dissemination of bacterial resistance within hospital settings include educational policies aimed at better adherence to infection control practices among professionals, particularly with regard to hand hygiene; and strategies for rationalizing the prescription of antimicrobials within hospitals. ${ }^{5-7}$

It has now become clear that the use of antimicrobials favors the emergence of resistant bacterial strains. However, it is also known that the genetic mechanism used by bacteria to acquire resistance to antibiotics not only promotes their spread within hospital environments but also confers stability on the resistance genes, even subsequently, in situations of absence of exposure to antimicrobials. ${ }^{8}$

This interaction between antibiotic consumption and the development of bacterial resistance to them is of particular interest with regard to Gram-negative bacilli (GNB). The resistance development pattern of GNB today provides a great challenge in treating infections caused by these bacteria. Given that new therapeutic options for treating infections caused by these GNB with high levels of resistance are not expected to become available in the near future, it becomes imperative to study measures that might be capable of improving the antimicrobial susceptibility of these bacterial agents, thereby making it possible to use the drugs that are available. Studies showing the effects of modifications to antibiotic prescription patterns are especially of interest in relation to combating the emergence of resistance in GNB strains. ${ }^{6}$ The objective of this study was to assess the impact of restriction of cefepime use on antimicrobial susceptibility among the Gram-negative bacilli (GNB) most frequently involved in healthcare-associated infections at a tertiary-level orthopedic hospital.

\section{Methods}

This observational study of an ecological nature was conducted at the Institute of Orthopedics and Traumatology, Hospital das Clínicas, University of São Paulo School of Medicine, which provides specialized high-complexity tertiary-level care within the field of orthopedics and traumatology in the city of São Paulo, Brazil. It was approved by the local Ethics Committee under the number 0649/07. At the time covered by this study, the hospital had an average of 150 beds available for operations, of which ten were for intensive care. In May 2007, the standard empirical antibiotic therapy for surgical site infections in this hospital was changed. This is the main type of hospital infection seen at this hospital, because of the attendance profile. Up to this date, combination therapy with vancomycin and cefepime was the first choice for empirical treatment of these infections until results had been obtained from cultures, and the consumption of both of these drugs was very high. After this date, a regime using teicoplanin and amikacin became the standard for empirical antibiotic therapy in such cases, and the use of cefepime was restricted. For the present study, data relating to hospital occupancy and mortality profiles; incidence of healthcare-associated infection cases; incidence of GNB as the cause of healthcare-associated infection; consumption of antimicrobials in the hospital (in defined daily doses [DDD]/1000 patient-days); and antimicrobial susceptibility for GNB relating to healthcare infection were obtained and compared between two periods: a 24-month period preceding the restriction on cefepime use, from May 2005 to May 2007 (first period); and a 24-month period subsequent to introducing the restriction, from May 2007 to May 2009 (second period).

For this study, the criteria for defining nosocomial infection recommended by the Centers for Disease Control and Prevention (CDC) in 2004 were used. ${ }^{10}$ For the analysis, only the GNB for which more than 30 isolates were obtained in each of the two study periods were selected, in accordance with the norms recommended by the Clinical and Laboratory Standards Institute (CLSI) for analysis and interpretation of cumulative antibiograms. ${ }^{11}$ The institutional policies for controlling healthcare-associated infections remained the same throughout the two periods. To meet the study objectives, the consumption of each antimicrobial was described separately and per class using summary measurements for each of the two periods (prior and subsequent to the restriction of cefepime use), and comparisons were made between the periods using the nonparametric Mann-Whitney test. ${ }^{12}$ Hospital occupancy and mortality measurements were described and compared in the same manner. Measurements of the antimicrobial susceptibility for each of the selected microorganisms were made according to the period, using absolute and relative frequencies, and the presence of associations in each period was investigated by means of the chi-square test, or by means of the Fisher exact test when the sample was very small. ${ }^{12}$ The tests were conducted using a significance level of 5\%.

\section{Results}

There were no differences between the two periods regarding the mean length of hospital stay or the mean hospital mortality rate, and there was no significant difference in the incidence of healthcare-associated infection cases between the two periods. The incidence of GNB as the cause of healthcare-associated infection also did not vary significantly between the two study periods (Table 1). 
Table I Comparison between the patients' mean length of hospital stay, and the mean hospital mortality rates, (defined as the mean $\mathrm{HAl}$ rates and the numbers of GNB isolates relating to $\mathrm{HAl}$ ), between the periods before and after restriction on cefepime use

\begin{tabular}{llll}
\hline & $\begin{array}{l}\text { 24-month period preceding } \\
\text { restriction on cefepime use }\end{array}$ & $\begin{array}{l}\text { 24-month period subsequent } \\
\text { to restriction on cefepime use }\end{array}$ & $\begin{array}{l}\text { P value } \\
\text { Mean length of hospital stay }\end{array}$ \\
Mean hospital mortality rate & 7.05 days & 6.88 days & 0.145 \\
Mean general HAl rate & $0.78 \%$ & $0.70 \%$ & 0.509 \\
Number of GNB isolates causing HAI & $3.78 \%$ & $3.73 \%$ & 0.984 \\
\hline
\end{tabular}

Abbreviations: HAl, healthcare-associated infections; GNB, Gram-negative bacilli.

With regard to antimicrobial consumption, there was a notable and significant drop in the consumption of cefepime after its use was restricted, and a significant increase in the consumption of amikacin. Other antibiotics that presented statistically significant increased consumption after cefepime use was restricted were aztreonam, ertapenem and levofloxacin. The consumption of imipenem and colistin was lower, in relation to the first study period, after cefepime use was restricted (Table 2).

The GNB with occurrences of more than 30 isolates (and for which antimicrobial susceptibilities were therefore analyzed) were the same in the two study periods: Acinetobacter baumanii, Pseudomonas aeruginosa, Klebsiella pneumoniae and Enterobacter spp. In the second period (after cefepime use was restricted), it was observed that the susceptibility for A. baumanii improved in relation to gentamicin and worsened in relation to imipenem. For $P$. aeruginosa, there was no change in antimicrobial susceptibilities after cefepime use was restricted. For K. pneumoniae and Enterobacter spp, there were improvements in the susceptibility relating to ciprofloxacin, after cefepime use was restricted. Table 3 shows comparisons of the antimicrobial susceptibilities for the GNB studied in the two study periods.

\section{Discussion}

The growing resistance to antimicrobials presented by the bacterial agents involved in healthcare-associated infections has been shown to be a very important problem over recent decades, particularly with regard to GNB. ${ }^{1,3}$ Strategies for rationalizing antimicrobial prescriptions within hospital environments and for changing the pattern of prescriptions for this class of drugs have been used as tools for combating the constant emergence of resistance among GNB. ${ }^{6}$

Analysis of the antimicrobial consumption data in the two study periods showed firstly that the policy of restriction of cefepime use that was instituted in the hospital was effective, considering that the mean monthly consumption of this antibiotic went down from 159.57 DDD per 1000 patient-days in the first period to 13.45 DDD per 1000 patient-days in the second period, and this change was statistically significant.
The observed significant increase in amikacin consumption in the second period, from a monthly mean of 13.03 DDD per 1000 patient-days to 93.79 DDD per 1000 patient-days can be explained by its role in the second study period as a standard antibiotic, replacing cefepime, for empirical treatment of surgical site infections. A drop in imipenem consumption was also observed after this restriction was implemented. This, in turn, correlated more with the increased consumption of ertapenem in the second study period than with the restriction on cefepime use. In the hospital where this study was conducted, ertapenem was introduced in March 2006 as the antibiotic of choice for treating infections caused by extended spectrum beta lactamase (ESBL)-producing Enterobacteriaceae, and the results from the impact of this introduction, particularly regarding the antimicrobial susceptibility of $P$. aeruginosa, have already been published. ${ }^{13-15}$ Analysis of the consumption of aztreonam, colistin and levofloxacin showed that although there was a statistically significant change between the two periods, the consumption of these drugs was very low throughout the study, compared with the consumption of other antibiotics, thus diminishing the importance of this finding. On the other hand, the consumption of ciprofloxacin went up from a monthly mean of 38.61 DDD per 1000 patient-days to $50.89 \mathrm{DDD}$ per 1000 patient-days after restriction of cefepime use. This increasing trend may be related to changes in the sensitivity of GNB in the second study period, since there were improvements in the antimicrobial susceptibilities of $K$. pneumoniae and Enterobacter spp in the period following the restriction of cefepime use.

For A. baumanii, the analysis of the antimicrobial susceptibility showed that there was a significant improvement in susceptibility in relation to gentamicin and worsening in relation to imipenem, although there were no differences in the consumption of these antimicrobials in the hospital. Despite the restriction on cefepime use, there was no change in the susceptibility of $A$. baumanii in relation to this antimicrobial. Regarding the susceptibility of P. aeruginosa, analysis and comparison of the data from the two study periods showed that there was no change after cefepime use was restricted, 
Table 2 Comparison of the antimicrobial susceptibilities among the main GNB species involved in nosocomial infections, between the periods preceding and subsequent to restriction on cefepime use

\begin{tabular}{|c|c|c|c|}
\hline $\begin{array}{l}\text { Pathogen/Antibiotic (number } \\
\text { of isolates tested before/after } \\
\text { restriction on cefepime use) }\end{array}$ & $\begin{array}{l}\text { Susceptibility (\%) in } 24-\text { month } \\
\text { period preceding restriction } \\
\text { on cefepime use }\end{array}$ & $\begin{array}{l}\text { Susceptibility (\%) in } 24 \text {-month } \\
\text { period subsequent to restriction } \\
\text { on cefepime use }\end{array}$ & $P$ value \\
\hline \multicolumn{4}{|l|}{ A. baumanii } \\
\hline Amikacin $(58 / 35)$ & 13.8 & 14.3 & $>0.999$ \\
\hline Ampicillin/Sulbactam (I I/37) & 90.9 & 78.4 & 0.662 \\
\hline Cefepime (50/35) & 6 & 8.6 & 0.687 \\
\hline Ceftazidime $(58 / 29)$ & 8.6 & 6.9 & $>0.999$ \\
\hline Ceftriaxone $(48 / 37)$ & 4.2 & 2.7 & $<0.999$ \\
\hline Ciprofloxacin (48/30) & 12.5 & 6.7 & 0.704 \\
\hline Gentamicin $(57 / 37)$ & 22.8 & 56.8 & 0.001 \\
\hline Imipenem (47/37) & 95.7 & 56.8 & $<0.001$ \\
\hline Trimethoprim-sulfamethoxazole (48/35) & 18.8 & 14.3 & 0.592 \\
\hline \multicolumn{4}{|l|}{ P. aeruginosa } \\
\hline Amikacin (44/42) & 75 & 66.7 & 0.395 \\
\hline Cefepime (30/4I) & 80 & 63.4 & 0.130 \\
\hline Ceftazidime $(44 / 4 \mathrm{I})$ & 61.4 & 65.9 & 0.667 \\
\hline Ciprofloxacin $(3 \mathrm{l} / 40)$ & 64.5 & 57.5 & 0.549 \\
\hline Gentamicin (43/4I) & 74.4 & 63.4 & 0.276 \\
\hline Imipenem (30/42) & 83.3 & 66.7 & 0.114 \\
\hline Piperacillin/Tazobactam (7/36) & 57.1 & 66.7 & 0.680 \\
\hline \multicolumn{4}{|l|}{ K. pneumoniae } \\
\hline Amikacin (39/29) & 84.6 & 86.2 & $>0.999$ \\
\hline Cefepime (20/29) & 25 & 31 & 0.646 \\
\hline Ceftazidime $(22 / 20)$ & 18.2 & 25 & 0.714 \\
\hline Cefotaxime (20/I5) & 15 & 33.3 & 0.246 \\
\hline Ceftriaxone (19/14) & 15.8 & 21.4 & $>0.999$ \\
\hline Ciprofloxacin (36/29) & 27.8 & 51.7 & 0.049 \\
\hline Gentamicin (39/29) & 23.1 & 34.5 & 0.300 \\
\hline Imipenem (22/29) & 100 & 100 & * \\
\hline Piperacillin/Tazobactam (3/20) & 33.3 & 20 & $>0.999$ \\
\hline Trimethoprim-sulfamethoxazole (39/29) & 46.2 & 62.1 & 0.193 \\
\hline \multicolumn{4}{|l|}{ Enterobacter spp } \\
\hline Amikacin $(32 / 37)$ & 78.1 & 86.5 & $0.36 \mathrm{I}$ \\
\hline Cefepime $(22 / 37)$ & 72.7 & 75.7 & 0.801 \\
\hline Ceftazidime $(26 / 32)$ & 69.2 & 78.1 & 0.442 \\
\hline Cefotaxime $(2 \mathrm{I} / 23)$ & 47.6 & 69.6 & 0.139 \\
\hline Ceftriaxone (10/14) & 50 & 48.1 & 0.421 \\
\hline Ciprofloxacin (27/37) & 48.1 & 73 & $<0.043$ \\
\hline Gentamicin $(31 / 37)$ & 83.9 & 83.8 & 0.992 \\
\hline Imipenem (27/37) & 100 & 100 & $*$ \\
\hline Trimethoprim-sulfamethoxazole $(32 / 37)$ & 32 & 37 & 0.227 \\
\hline
\end{tabular}

Note: " $P$ values not able to be calculated because there were no cases of resistance. Abbreviation: GNB, Gram-negative bacilli.

for any of the antibiotics tested. For K. pneumoniae and Enterobacter spp, comparison of the susceptibilities data showed that there were significant improvements in the susceptibilities of these bacterial agents in relation to ciprofloxacin, in the second study period, although the consumption of this antimicrobial increased in this period. Comparison between these results and those from previous studies, regarding the impact of changes in antimicrobial prescriptions on the sensitivity profile of GNB involved in hospital infections shows the complexity of this interaction, given that changes in the use of a single class of antimicrobials may produce different changes in relation to the susceptibilities of the bacterial agents studied. ${ }^{6,16-19}$

Between the two study periods, there was no difference in the patients' mean length of hospital stay or in the hospital mortality rate. Likewise, there was no significant difference in the nosocomial infection incidences. These data make it possible to infer indirectly that the severity of the hospitalized 
Table 3 Comparison of mean monthly consumption, in DDD/1000 patient-days, of antimicrobials with a spectrum of action directed against GNB, between the periods preceding and subsequent to restriction of cefepime use

\begin{tabular}{|c|c|c|c|}
\hline Antimicrobial & $\begin{array}{l}\text { Mean monthly consumption } \\
\text { in the } 24-m o n t h \text { period preceding } \\
\text { restriction on cefepime use }\end{array}$ & $\begin{array}{l}\text { Mean monthly consumption } \\
\text { in the } 24 \text {-hour period subsequent } \\
\text { to restriction on cefepime use }\end{array}$ & $P$ value \\
\hline Amikacin & 13.03 & 93.79 & $<0.001$ \\
\hline Ampicillin/Sulbactam & 76.62 & 66.64 & 0.156 \\
\hline Aztreonam & 0.44 & 0.78 & 0.005 \\
\hline Cefepime & 159.57 & 13.45 & $<0.001$ \\
\hline Ceftriaxone & 57.86 & 50.47 & 0.119 \\
\hline Ceftazidime & 28.59 & 25.90 & 0.968 \\
\hline Ciprofloxacin & 38.61 & 50.89 & 0.075 \\
\hline Colistin & 0.86 & 0.01 & $<0.001$ \\
\hline Ertapenem & 10.05 & 20.50 & 0.003 \\
\hline Gentamicin & 68.24 & 61.38 & 0.298 \\
\hline Imipenem & 36.53 & 15.02 & $<0.001$ \\
\hline Levofloxacin & 1.29 & 2.73 & 0.020 \\
\hline Meropenem & 14.76 & 17.56 & 0.589 \\
\hline Piperacillin/Tazobactam & 1.46 & 2.15 & 0.136 \\
\hline Polymyxin B & 0.07 & 0.03 & 0.555 \\
\hline
\end{tabular}

Abbreviations: GNB, Gram-negative bacilli; DDD/I000, defined daily dose per thousand.

patients' conditions was probably similar between the two periods, and that the prevalence of nosocomial infection among the hospitalized patients was also similar between the two periods. This is important because differences in the severity of patients' conditions or in the prevalence of nosocomial infection among patients may influence the variations in bacterial susceptibilities described above.

The limitations of ecological studies for analyzing relationships between antimicrobial consumption and the development of bacterial resistance within hospital environments have already been explored..$^{20}$ Bacterial resistance is directed by the principles of natural selection, and its underlying selection and dispersion mechanisms are matters of great complexity. The influence of antimicrobial consumption on this resistance may even have different effects over the course of time, and will be most important at the time when a specific resistance mechanism appears. Regarding the analysis of bacterial susceptibilities, the cutoff points established by the CLSI for defining resistance, as used in these types of study, are very high and exclude bacterial strains with low resistance levels from the analysis. Such strains usually precede strains with high resistance levels and would also be important in analyses of hospital environments. ${ }^{21}$

One limitation in particular in this study was the absence of molecular biology analysis of the bacteria involved, which would have made it possible to evaluate whether the observed differences in antimicrobial susceptibility were caused by the introduction of new bacterial strains or by clonal expansion. Another deficiency was the lack of measurements on healthcare professionals' adherence to hospital infection control practices in the two study periods. Although the recommendations for such practices remained the same throughout the two periods, differences in adherence to the recommendations may have influenced the results obtained.

The results from this study suggest that the restriction on cefepime use had a positive impact on the sensitivity profiles of the K. pneumoniae and Enterobacter spp strains involved in healthcare-associated infections at the orthopedic hospital where this study was conducted, given that after the restriction was introduced, the susceptibility to ciprofloxacin improved. However, for A. baumanii, the results suggest that there was a negative impact, given the worsening of susceptibility to imipenem.

\section{Acknowledgment}

The authors would like to thank Dr Silvia F Costa for her careful review of this work.

\section{Disclosure}

The authors report no conflict of interest in this work.

\section{References}

1. Bartoloni A, Bartalesi F, Mantella A, et al. High prevalence of acquired antimicrobial resistance unrelated to heavy antimicrobial consumption. J Infect Dis. 2004;189(7):1291-1294.

2. Rahal JJ, Urban C, Segal-Maurer S. Nosocomial antibiotic resistance in multiple Gram-negative species: experience at one hospital with squeezing the resistance balloon at multiple sites. Clin Infect Dis. 2002; 34(4):499-503. 
3. Lautenbach E. Antimicrobial resistance in Gram-negative pathogens: crafting the tools necessary to navigate the long ascent out of the abyss. J Infect Dis. 2009;200(6):838-840.

4. van de Sande-Bruinsma N, Grundmann H, Verloo D, et al. Antimicrobial drug use and resistance in Europe. Emerg Infect Dis. 2008;14(11): $1722-1730$.

5. Drummond CW. Resistance is futile - a conference to promote the rational use of antimicrobials in acute hospitals. J Antimicrob Chemother. 2006;57(2):171-175.

6. Ntagiopoulos PG, Paramythiotou E, Antoniadou A, Giamarellou H, Karabinis A. Impact of an antibiotic restriction policy on the antibiotic resistance patterns of Gram-negative microorganisms in an intensive care unit in Greece. Int J Antimicrob Agents. 2007;30(4):360-365.

7. de Araujo OR, da Silva DC, Diegues AR, et al. Cefepime restriction improves Gram-negative overall resistance patterns in neonatal intensive care unit. Braz J Infect Dis. 2007;11(2):277-280.

8. Burke JP. Antibiotic resistance - squeezing the balloon? JAMA. 1998; 280(14):1270-1271.

9. Livermore D. Has the era of untreatable infections arrived? JAntimicrob Chemother. 2009;64(Suppl 1):i29-i36.

10. Horan TC, Gaynes RP. Surveillance of nosocomial infections. In: Mayhall CG, editor. Hospital Epidemiology and Infection Control, 3rd ed. Philadelphia, PA: Lippincott Williams and Wilkins; 2004: 1659-1702.

11. Clinical and Laboratory Standards Institute. Analysis and Presentation of Cumulative Antimicrobial Susceptibility Test Data: Approved Guideline-3rd ed. CLSI document M39-A3. Wayne, PA: Clinical and Laboratory Standards Institute; 2009.

12. Kirkwood BR, Sterne JA. Essential Medical Statistics. 2nd ed. Malden, MA: Blackwell Science; 2003:502.
13. Lima AL, Oliveira PR, Paula AP, Zumiotti AV. Influence of ertapenem administration on the incidence of carbapenem-resistant Pseudomonas aeruginosa. Braz J Infect Dis. 2008;12(2):105-106.

14. Lima AL, Oliveira PR, Paula AP, Dal-Paz K, Rossi F, Zumiotti AV. The impact of ertapenem use on the susceptibility of Pseudomonas aeruginosa to imipenem: a hospital case study. Infect Control Hosp Epidemiol. 2009;30(5):487-490.

15. Lima AL, Oliveira PR, Paula AP, et al. Carbapenem stewardship: positive impact on hospital ecology. Braz J Infect Dis. 2011;15(1):1-5.

16. Du B, Chen D, Liu D, et al. Restriction of third-generation cephalosporin use decreases infection-related mortality. Crit Care Med. 2003; 31(4):1088-1093.

17. Brahmi N, Blel Y, Kouraichi N, et al. Impact of ceftazidime restriction on Gram-negative bacterial resistance in an intensive care unit. J Infect Chemother. 2006;12(4):190-194.

18. Meyer E, Lapatschek M, Bechtold A, Schwarzkopf G, Gastmeier P, Schwab F. Impact of restriction of third generation cephalosporins on the burden of third generation cephalosporin resistant $K$. pneumoniae and E. coli in an ICU. Intensive Care Med. 2009;35(5):862-870.

19. Rahal JJ, Urban C, Horn D, et al. Class restriction of cephalosporin use to control total cephalosporin resistance in nosocomial Klebsiella. JAMA. 1998;280(14):1233-1237.

20. Cantón R, Cobo J. Hospital antibiotic use and resistance: a relationship that is difficult to measure and complex to interpret. Enferm Infecc Microbiol Clin. 2009;27(8):437-440. [Spanish.]

21. Bisson G, Fishman NO, Patel JB, Edelstein PH, Lautenbach E. Extended-spectrum beta-lactamase-producing Escherichia coli and Klebsiella species: risk factors for colonization and impact of antimicrobial formulary interventions on colonization prevalence. Infect Control Hosp Epidemiol. 2002;23(5):254-260.
Infection and Drug Resistance

\section{Publish your work in this journal}

Infection and Drug Resistance is an international, peer-reviewed openaccess journal that focuses on the optimal treatment of infection (bacterial, fungal and viral) and the development and institution of preventive strategies to minimize the development and spread of resistance. The journal is specifically concerned with the epidemiology of antibiotic

\section{Dovepress}

resistance and the mechanisms of resistance development and diffusion in both hospitals and the community. The manuscript management system is completely online and includes a very quick and fair peerreview system, which is all easy to use. Visit http://www.dovepress.com/ testimonials.php to read real quotes from published authors. 